\title{
High Sensitive Photopolymerization Initiator System using Violet Laser and Its Application to Photopolymer CTP Plate
}

\author{
Akinori Shibuya, Kazuto Kunita and Shigeo Koizumi \\ Synthetic Organic Chemistry Laboratories, $R \& D$ Management Headquarters, \\ FUJIFILM Corporation \\ 4000 Kawashiri Yoshida-cho Haibara-gun Shizuoka, 421-0396, Japan \\ TEL: +81-548-34-5363, FAX: +81-548-32-3919 \\ akinori_shibuya@yoshis.fujifilm.co.jp
}

\begin{abstract}
A highly sensitive photopolymerization system by using violet laser, $405 \mathrm{~nm}$, was investigated. The photopolymerization composition which contained an initiator and a high fluorescent dye showed efficient sensitization of the system. The photosensitization mechanism seemed to have a singlet energy transfer process. A key molecular design of the dye getting the higher photosensitization efficiency is a repression of thermal relaxation from the excitation state. The photopolymerization system was applied to the computer to plate (CTP) technology, a digital printing plate, to develop our i-Presso P-NV for the violet laser. As compared with a system using a conventional FD-YAG laser, the P-NV system revealed the advantage of high productivity on plate making and also handling under a brighter safelight.
\end{abstract}

Keywords: highly sensitive photopolymerization system, fluorescent dye, violet laser

\section{Introduction}

An important technical challenge in the printing industry is to develop a lithographic printing plate that can be used in the CTP (computer-to-plate) system, which uses digitalized image information to manipulate a laser beam and directly produce a printing plate[1,2]. One proposed example is a system based on a photopolymer CTP plate. This system records image on a photopolymerizable material that possesses an excellent photosensitization speed, using FD-YAG laser (emission wavelength $=532 \mathrm{~nm}$ ) [3]. However, development of a system that is more low-cost, improved in productivity by virtue of a higher writing speed, and easier to handle (i.e. usable under a brighter yellow safelight) is desired in the field.

On the other hand, an InGaN violet laser with an emission wavelength of $405 \mathrm{~nm}$ has recently became available, bringing the possibility to develop a novel photopolymer CTP plate system that can meet the above requirements. In this situation, the enhanced low cost/high productivity as described above is achievable by developing a smaller and faster spinner mirror for a low-cost laser and by increasing the drawing speed using a shorter laser wavelength. Finally the easiness to handle can be obtained by designing a photosensitive material that is compatible with a shorter laser wavelength and thus is not sensitive to yellow light.

In order to realize such a system, however, it is required to develop a photopolymerization initiation system that consists of a novel photopolymerization initiator and a sensitizing dye having an improved sensitivity to violet laser.

We have made an intensive study and successfully discovered a violet laser-compatible, highly sensitive photopolymerization initiation system that is superior in light absorption efficiency and sensitization efficiency. We further applied our photopolymerization initiation system to photopolymer CTP plate and developed a violet laser-compatible photopolymer CTP plate (i-Presso P-NV), which is low-cost, highly productive, and easy to handle, as described below.

In addition, highly sensitive photopolymerization initiation systems[4,5] are strongly desired technologies which are widely useful in the fields

$\begin{array}{llr}\text { Received } & \text { April } & 1,2013 \\ \text { Accepted } & \text { May } & 10,2013\end{array}$


such as: the imaging materials field for laser lithography, holography, and the like; the electronic materials field for photoresists and the like; and the light curable resin materials field for inks, coatings, adhesives, and the like[6,7].

\section{Experimental}

\subsection{Calculation of Excitation Energy}

Electronic absorption spectra and fluorescence spectra of sensitizing dyes were normalized and the intersection point was determined. The energy for the wavelength corresponding to the intersection point, which was defined as the excitation energy, was calculated. Electronic absorption spectra were measured as transmission spectra in a THF solvent, using a UV-Vis-NIR Spectrophotometer Cary5G (manufactured by Varian). For fluorescence spectra measurement, we produced polymer matrices each containing any one of the sensitizing dyes and used a SPEX Fluorolog-3 fluorescence spectrophotometer (manufactured by JOBIN YVON) in the photoncounting mode.

\subsection{Calculation of Oxidation Potential $\left(\right.$ Eox $\left.^{*}\right)$ in} Excited Singlet State

Using potentiometers

(GPIB

POTENTIOSTAT/GALVANOSTAT HA-501G and ARBITRARY FUNCTION GENERATOR HB-105, manufactured by Hokuto Denko, Co.), we measured oxidation potentials in acetonitrile supplemented with tetrabutylammonium perchlorate as an electrolyte, using an $\mathrm{Ag} / \mathrm{AgCl}$ reference electrode and a $\mathrm{Pt}$ counter electrode. Oxidation potential in an excited singlet state was defined as follows.

Oxidation Potential in Excited Singlet State = Measured Oxidation Potential - Excitation Energy as Described Above.

\subsection{Evaluation of Sensitivity}

A photopolymerizable material was prepared by (i) spreading a photosensitizing resin that consists of a photopolymerization initiator, a sensitizing dye, a polymerizable polyfunctional monomer, an alkali-soluble binder, and a pigment on a hydrophilized aluminum support and then (ii) covering the photosensitizing resin with a PVAbased oxygen insulation layer. A Fuji Step Guide manufactured by Fujifilm (discontinuous transmittance optical densities $(\Delta \mathrm{D}=0.15)$, gray scale) was adhered to the resultant photosensitive material. The exposure was performed at predetermined exposure energy levels using an optical filter and a Xenon lamp. For the purpose of estimating the exposure aptitude for short wave semiconductor laser, exposure was carried out with a monochromic light of $400 \mathrm{~nm}$ using Kenko BP40 as an optical filter. Thereafter, development was performed by immersing the exposed photopolymerizable material in an alkaline developer $(\mathrm{pH} 12.0)$ at $25^{\circ} \mathrm{C}$ for 10 seconds, the exposure energy was measured, and the sensitivity (unit $=\mu \mathrm{J} / \mathrm{cm}^{2}$ ) was calculated from the highest step number where the image was completely removed.

\subsection{Insolubilization Ratio of Lith Film}

A photosensitive material prepared in the same fashion as described in the Evaluation of Sensitivity section was subjected to exposure using Kenko BP-40 as an optical filter. After the photosensitive material was heated at $120^{\circ} \mathrm{C}$ for 20 sec, it was immersed in an acetone/DMSO solvent for 2 hours. Its weight reduction ratio was measured after the immersion and used as the insolubilization ratio of the lith film.

\section{Results and Discussion}

3.1. Layer Configuration and Photosensitive Layer Materials

The highly sensitive photopolymerization initiation system of the present study can be applied to photopolymer CTP plates, in which radicals are generated by exposure to a violet laser and react with polyfunctional monomers to polymerize and cure (Fig.1). The photosensitive layer configuration and photosensitive layer materials shown in Table 1 was used for the present study.

Our technical objectives were to (i) control the photosensitive wavelength of photopolymerization photosensitive layer for efficient absorption of violet laser light (the spectral sensitivity should be around $405 \mathrm{~nm}$ for the sake of laser source compatibility) and (ii) optimize the photosensitization process (the sensitivity should allow a low-power $30 \mathrm{~mW}$ laser to generate sufficient radicals: $60-90 \mu \mathrm{J} / \mathrm{cm}^{2}$ ). The results are described below in detail.

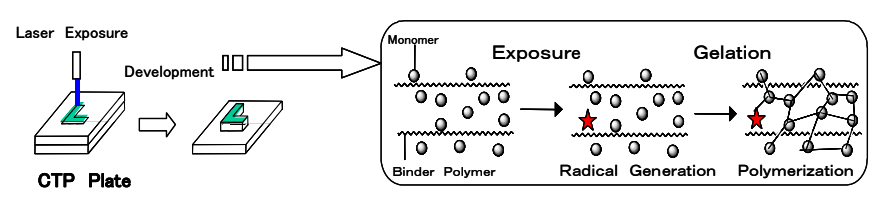

Figure 1. Process of image formation 
Table 1. Composition and material of photo sensitive layer

\begin{tabular}{|c|}
\hline Over Coat Layer \\
\hline Photopolymerization Layer \\
{$\left[\begin{array}{l}\text { Photopolymerization Initiator } \\
\text { Photo Sensitizing Dye } \\
\text { Photopolymerization Monomer } \\
\text { Alkali Soluble Binder Polymer } \\
\text { Pigment }\end{array}\right]$} \\
\hline Aluminium Support \\
\hline
\end{tabular}

3.2. Violet Laser-Compatible, Highly Sensitive Photopolymerization Initiation System

\subsubsection{Radical Generation Process}

In the present study, we focused on the binary photopolymerization initiation system, which contains a sensitizing dye responsible for light absorption/photosensitization process and a photopolymerization initiator for radical generation. A general concept of the radical generation process in the binary photopolymerization initiation system[8] (shown in Fig.2) involves (i) a light absorption process where a sensitizing dye absorbs $405 \mathrm{~nm}$ laser light and is activated to an excited singlet state and (ii) an initiator radical generation process based on the interaction between excited dye and photopolymerization initiator (photosensitization). Sensitizing dye has a crucial role in each process. To resolve these technical problems, we set out to design a "violet laser-compatible, highly sensitive sensitizing dye" by optimizing the $405 \mathrm{~nm}$ laser light absorption and the photosensitization process of sensitizing dyes.

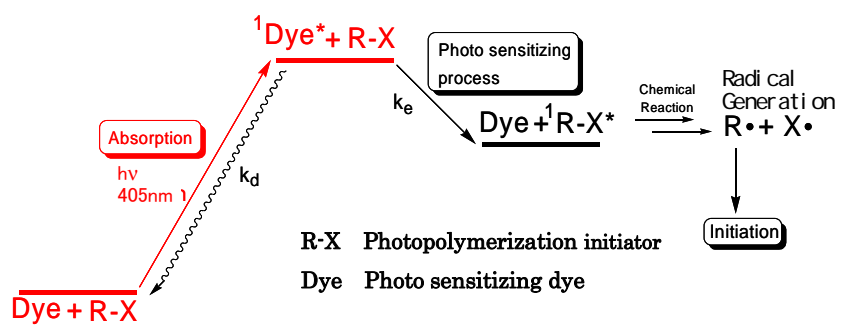

Figure 2. Radical generation process of photo initiator

\subsubsection{Optimization of Light Absorption}

Because a sensitizing dye of our interest should have an absorption wavelength compatible with the laser source, we searched for a sensitizing dye having an absorption wavelength of $400 \mathrm{~nm}$. We examined the absorption wavelength of Styryl, Merocyanine, Coumarin, and various other sensitizing dyes (structures for some of the sensitizing dyes are shown in Fig.3) and found that
Styryl and Merocyanine sensitizing dyes were superior in light absorption efficiency (Fig.4).

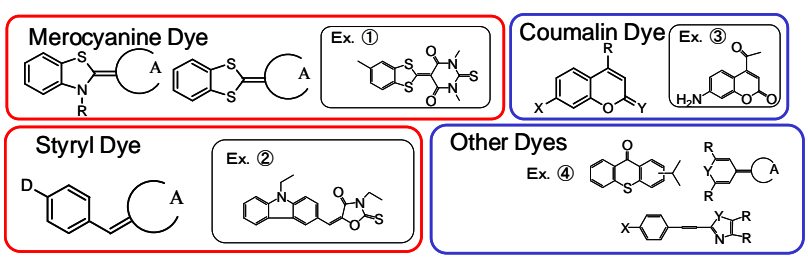

Figure 3. Example of photo sensitizing dyes

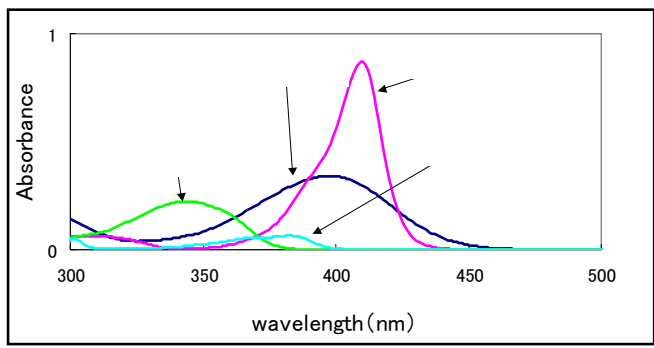

Figure 4. Absorption spectrum of various photo sensitizing dyes in THF solution:concentration $1.00 \times$ $10^{-5} \mathrm{M}$

We also examined the polymerization sensitivity of these sensitizing dye to violet laser. The initiator was a commercially available titanocene compound. As the result, Styryl and Merocyanine sensitizing dyes, which have good light absorption efficiencies, proved to be superior in polymerization sensitivity (Fig.5).
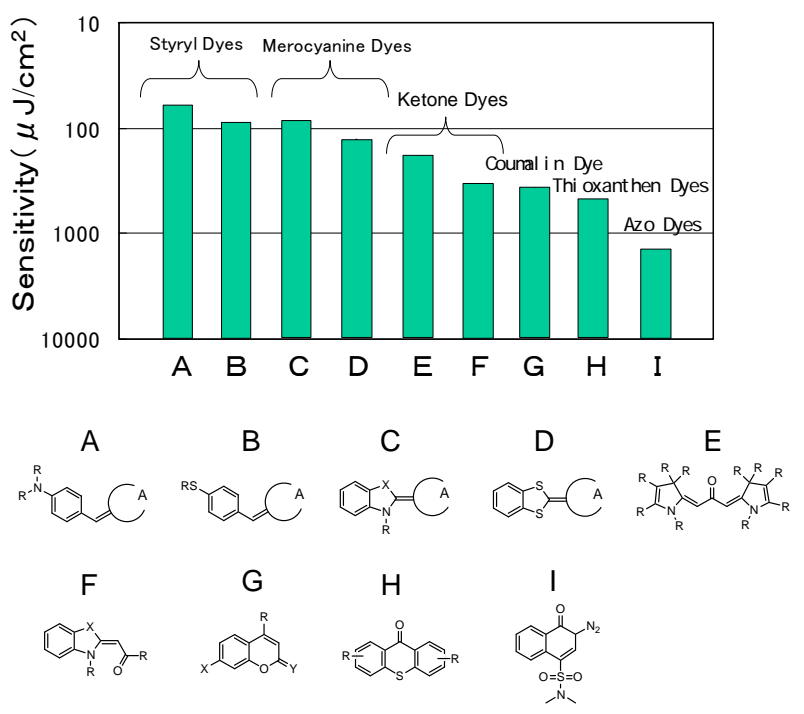

Figure 5. Sensitivity of photo sensitizing dyes

Among these dyes, the most sensitive Aminostyryl sensitizing dye was chosen for further optimization of the photosensitization process. 


\subsubsection{Optimization of Photosensitization Process}

The photosensitization process in this photopolymerization initiation system might be based on electron transfer and energy transfer reactions. However, our analytical experiment suggested that an excited singlet energy transfer from the excited singlet sensitizing dye to the photopolymerization initiator was crucial. Some of the experimental evidence is shown in Figs. 6 and 7.

(1) Correlation between Excitation Energy and Sensitivity

The oxidation potential of a dye in an excited singlet state (excited oxidation potential Eox ${ }^{*}$ hereinafter) is an index of its electron transfer sensitization efficiency. We found that excitation energy correlated with sensitivity in different dyes having similar Eox ${ }^{*}$ (Fig.6). That is, a higher excitation energy means a higher sensitivity.

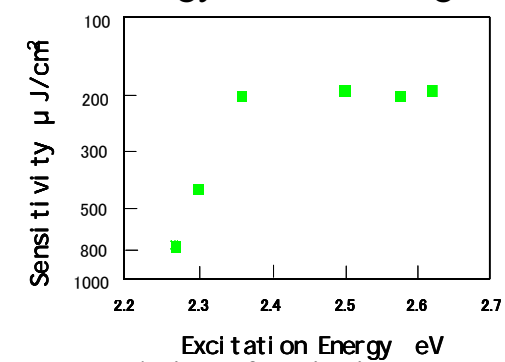

Figure 6. Correlation of excitation energy and sensitivity

(2) Correlation between Excited Singlet State Oxidation Potential (Eox*)and Sensitivity

We did not find a correlation between the excited oxidation potential Eox ${ }^{*}$ and the sensitivity of the dyes examined (Fig.7). In fact, a sensitizing dye that has a smaller absolute value of Eox ${ }^{*}$ (i.e., a less-electron-donating dye with low electron transfer efficiencv) was more sensitive.

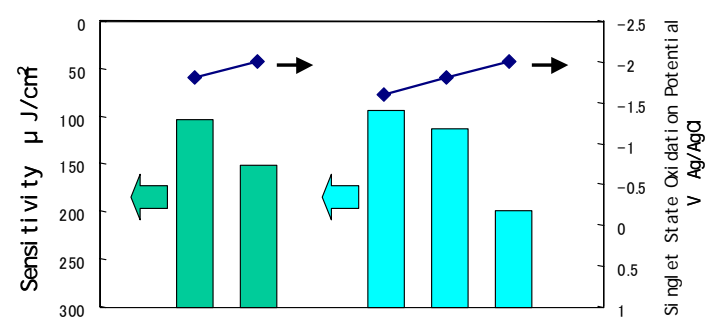

Figure 7. Correlation of singlet state oxidation potential and sensitivity

We took these results into consideration to design a sensitizing dye that exhibits an optimized excited singlet energy transfer. In photochemistry, the efficiency of energy transfer is known to correlate with the overlap integral of the emission spectrum of sensitizing dye and the absorption spectrum of initiator, and to be the higher the larger the overlap is ${ }^{8)}$. Thus it is more important to improve the emission intensity of a sensitizing dye than its other optical properties (Scheme 1 in Fig. 8: Image of the overlap integral in an energy transfer reaction based on electron exchange). We used the Aminostyryl sensitizing dye described in 3.2.2., which has a good absorption property/polymerization sensitivity, as a lead compound to design a molecule having an enhanced fluorescence

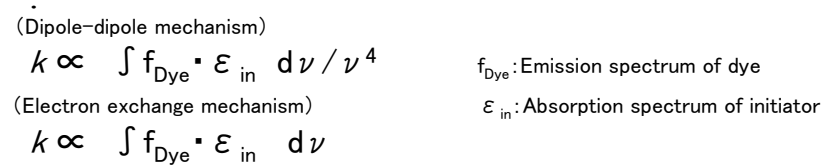

Scheme 1. Kinetic constant of energy transfer (k)

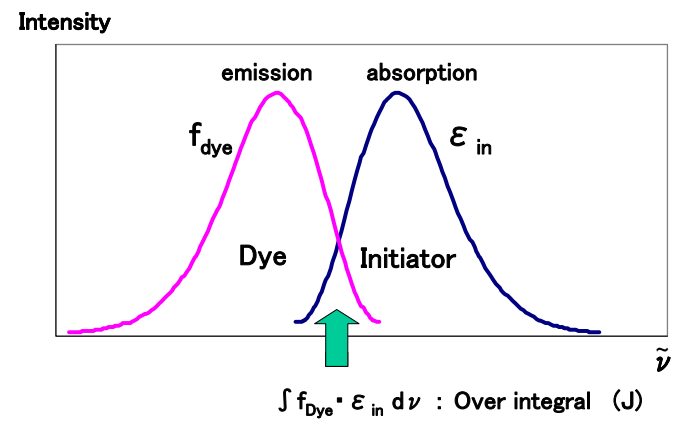

Figure 8. Absorption and Emission spectrum showing the overlap region and the corresponding overlap integral $(\mathrm{J})$

Fluorescence quantum yield can be calculated from rate constant of fluorescence $\left(\mathrm{k}_{\mathrm{f}}\right)$, rate constant of thermal quenching $\left(\mathrm{k}_{\mathrm{d}}\right)$, and rate constant of intersystem crossing $\left(\mathrm{k}_{\text {isc }}\right)$ (Scheme 2). It is critical to improve $\mathrm{k}_{\mathrm{f}}$ and/or suppress $\mathrm{k}_{\mathrm{d}}$ and $\mathrm{k}_{\text {isc }}$ in order to design a high fluorescence (Fig.9).

First, in order to suppress the $k_{d}$ (the thermal quenching), we tried to rigidify the molecular structure to prevent the rotation around the methine chain. This strategy resulted in an insufficient emission intensity improved only by 1.1 times.

Next, in order to improve the $\mathrm{k}_{\mathrm{f}}$, we performed a search for an acidic nucleus/basic nucleus that can enhance the oscillator strength, i.e. optimize the HOMO-LUMO electronic state. Enhancement of emission intensity of Styryl sensitizing dyes seemed difficult because these had a methine chain of easily rotatable structure. However, we successfully obtained a novel sensitizing dye 
having an emission intensity 5 times as high as that of the lead compound (Fig.10).

$$
\phi_{\mathbf{f}} \propto \frac{\mathbf{k}_{\mathbf{f}}}{\mathbf{k}_{\mathbf{f}}+\mathbf{k}_{\mathbf{d}}+\mathbf{k}_{\text {isc }}} \quad \begin{aligned}
& \phi_{\mathrm{f}}: \text { Fluorescence quantum yield } \\
& \mathrm{k}_{\mathrm{f}}: \text { Rate constant of fluorescence } \\
& \mathrm{k}_{\mathrm{d}}: \text { Rate constant of radationless transition } \\
& \mathrm{k}_{\text {isc }}: \text { Rate constant of intersystem crossing }
\end{aligned}
$$

Scheme 2. Expression of fluorescence quantum yield

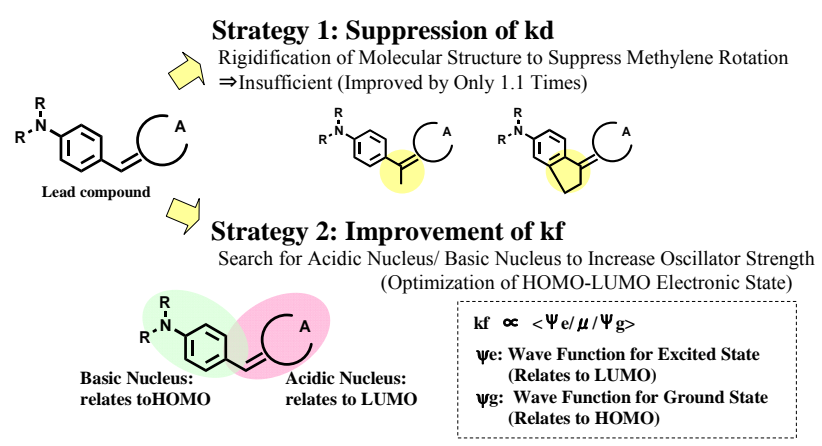

Figure 9. Molecular design of high fluorescent dye

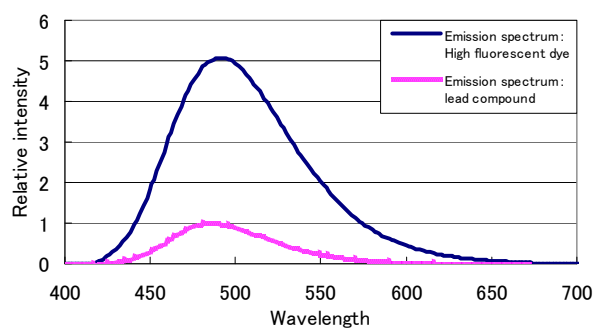

Figure 10. Fluorescence emission spectrum of photo sensitizing dye

We investigated possible reasons for the high fluorescence of the novel sensitizing dye. First, we compared the novel sensitizing dye and the lead compound for their oscillator strength, which correlates with kf. The two dyes had similar oscillator strength, indicating that the improved fluorescence was not due to improvement of the kf. We examined other possibilities by performing a molecular orbital calculation for model compounds (AM1 calculation). The result suggested that the electron density on the LUMO of the sensitizing dye is uniformly distributed over the methine chain, leading to a marked increase in the double-bond nature of $a-b$ in the excited state (Fig.11 and Table 2). These results suggest that our novel sensitizing dye has a high radiance possibly because its thermal quenching can be suppressed by an increase in the $\pi$ orbital rigidity in the excited state.
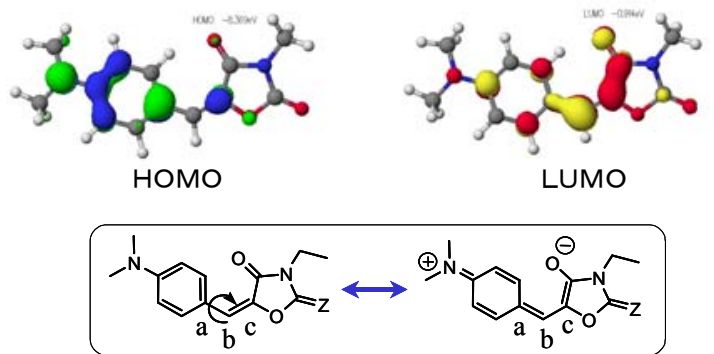

Figure 11. Molecular orbital calculation (AM1) of high fluorescent dye

Table 2. Bond order of high fluorescent dye

\begin{tabular}{|l|c|c|c|c|}
\hline \multirow{2}{*}{ Bond order } & \multicolumn{2}{|c|}{ HOMO } & \multicolumn{2}{c|}{ LUMO } \\
\cline { 2 - 5 } & $\mathrm{Ca}-\mathrm{Cb}$ & $\mathrm{Cb}-\mathrm{Cc}$ & $\mathrm{Ca}-\mathrm{Cb}$ & $\mathrm{Cb}-\mathrm{Cc}$ \\
\hline Lead compound & 1.06 & 1.75 & 1.12 & 1.60 \\
\hline $\begin{array}{l}\text { High fluorescent } \\
\text { Dye }\end{array}$ & 1.06 & 1.78 & 1.21 & 1.56 \\
\hline
\end{tabular}

We draw a Perrin plot for this sensitizing dye, which has a high radiance, combined with a titanocene compound as a quencher. This revealed that the quenching radius was relatively large, i.e. $17 \AA$ (Fig. 12). In addition, the fluorescence quenching rate was as high as about $70 \%$, suggesting its efficient energy transfer reaction.

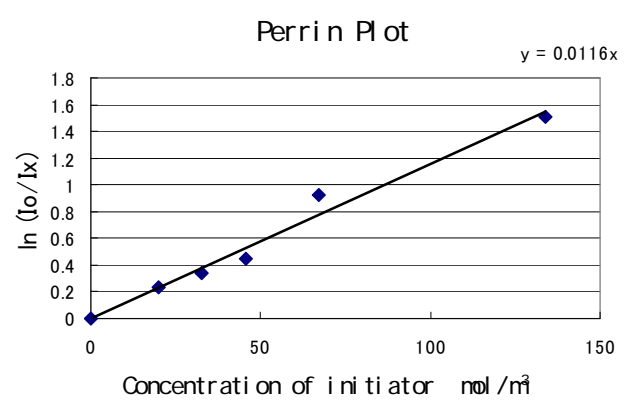

Figure 12. Perrin plot of high fluorescent dye

We further produced a photosensitive layer which include our photopolymerization initiation system incorporated in a polyfunctional monomer/binder polymer matrix. We measured its insolubilization ratio after exposure to a violet laser (Fig. 13). The insolubilization ratio of the photosensitive layer was as high as 60 to $70 \%$ in the practical exposure energy range for violet laser (50 to $100 \mu \mathrm{J} / \mathrm{cm}^{2}$ ), indicating that the lith film sufficiently polymerized and cured. 


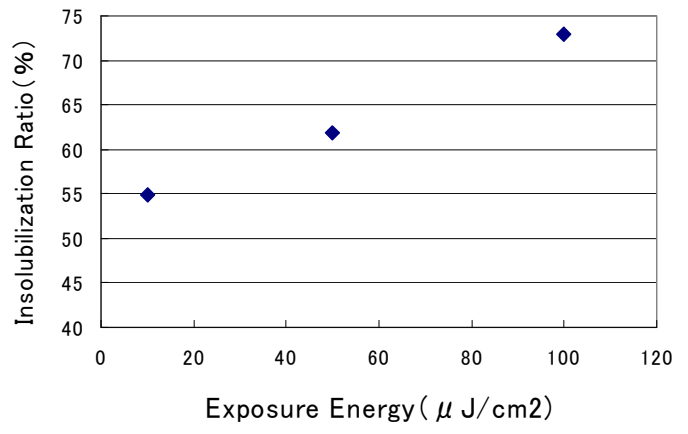

Figure 13. Insolubilization ratio of high fluorescent aye

\subsection{Application to Photopolymer CTP Plate (P- $\mathrm{NV}$ )}

The next challenge was to use our violet lasercompatible, highly sensitive photopolymerization initiation reaction for developing a highly sensitive photopolymer CTP plate. Because LP-NX, a conventional FD-YAG laser $(200 \mathrm{~mW}, 532 \mathrm{~nm})-$ compatible photopolymer CTP plate, needs to be handled under a red safelight, a sensitivity that allows easier handling has been desired. The reaction as described above can provide a novel system that uses an $\mathrm{InGaN}$ violet laser $(30 \mathrm{~mW}$, $405 \mathrm{~nm}$ ) instead of the FD-TAG laser and is thus usable under a yellow safelight, more low-cost, and highly productive.

By applying our violet laser-compatible, highly sensitive photopolymerization initiation system to photopolymer CTP plate, we successfully developed a superior photopolymer CTP plate (iPresso P-NV (P-NV hereinafter)) that has a spectroscopic sensitivity to a wavelength $100 \mathrm{~nm}$ shorter and is 2 to 3 times more sensitive, lowcost, highly productive, and easier to handle, compared to LP-NX[9,10]. The spectroscopic sensitivity and characteristic curve of P-NV are shown in Figs. 14 and 15 and its major properties are summarized in Table 3.

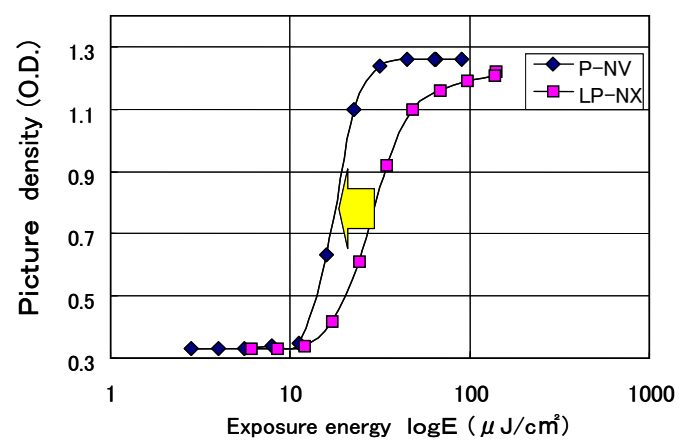

Figure 14. Characteristic Curve of P-NV

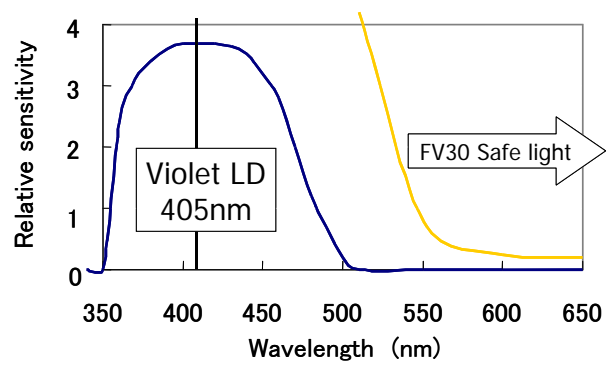

Figure 15. Spectroscopic Sensitivity of P-NV

Table 3. Main performance of P-NV

\section{Safe light: Orangeyellow Light "FV30"}

Light source: Violet Laser(405nm, 30mW)

Sensitivity: 60 to $90 \mu \mathrm{J} / \mathrm{cm}^{2}$

Resolution: 200 lines 2 to $98 \%$

Press Life: 200000 sheets(UVink 100000sheets)

\section{Conclusion}

By light absorption optimization and photosensitization process optimization, we successfully developed a violet laser-compatible, highly sensitive photopolymerization initiation system based on a novel sensitizing dye having a high radiance. We further applied this highly sensitive photopolymerization initiation system to photopolymer CTP plate and achieved a violet laser-compatible photopolymer CTP plate (iPresso P-NV), which is inexpensive, highly productive, and easy to handle compared to conventional photopolymer CTP plates.

\section{References}

1. S. Kondo: Journal of Printing Science and Technology, 41 (2004) 48.

2. Y. Urabe, Japan Printer (Insatsu Zasshi),87 (2004),3.

3. S. Kondo, Engineering Materials (KogyoZairyo),48 (2000) 54.

4. B. M. Monroe, Chemical Review,93 (1993) 435.

5. R. S. Davidson, Journal of Photochemistry and Photobiology A:Chemistry,73 (1993) 81.

6. J. P. Faussie, Rapra Rev. Rep, 9 (1998) 1-149.

7. M. Tsunooka et al, Prog.Polym.Sci.,21 (1996) 1.

8. Gilbert \& Baggot, :Essentials of Molecular Photochemistry (1991).

9. A. Shibuya, S. Koizumi, and K. Kunita, Japanese Society of Printing Science and Technology 112th Spring Meeting Abstract, p.56 (2004).

10. A. Shibuya, S. Koizumi, \& K. Kunita, Journal of Printing Science and Technology, 42 (2005) 28. 